in vivo $34: 1527-1531(2020)$

doi:10.21873/invivo.11941

\title{
Debulking Surgery for Moderately Differentiated Neuroendocrine Gastric Carcinoma - A Case Report and Literature Review
}

\author{
NICOLAE BACALBASA ${ }^{1,2,3}$, IULIANA HALMACIU ${ }^{4}$, CIPRIAN BOLCA $^{5}$, ADRIAN NEACSU ${ }^{1,6}$, \\ DRAGOS CRETOIU ${ }^{1,7}$, CRISTIAN BALALAU $^{1,8}$, CAMELIA DIACONU ${ }^{1,9}$, \\ LAURA ILIESCU ${ }^{1,10}$, ALEXANDRU FILIPESCU ${ }^{1,11}$, CORA POP $^{1,9}$ and IRINA BALESCU ${ }^{12}$ \\ 1 "Carol Davila" University of Medicine and Pharmacy, Bucharest, Romania; \\ ${ }^{2}$ Department of Obstetrics and Gynecology, "I. Cantacuzino" Clinical Hospital, Bucharest, Romania; \\ ${ }^{3}$ Department of Visceral Surgery, Center of Excellence in Translational \\ Medicine "Fundeni" Clinical Institute, Bucharest, Romania; \\ ${ }^{4}$ Department of Anatomy, "George Emil Palade" University of Medicine, \\ Pharmacy, Science and Technology of Târgu Mureș, Târgu Mureș, Romania; \\ ${ }^{5}$ Department of Thoracic Surgery, "Marius Nasta" Institute of Pneumonology, Bucharest, Romania; \\ ${ }^{6}$ Department of Obstetrics and Gynecology, "St. John" Emergency Clinical Hospital, Bucharest, Romania; \\ 7 "Alessandrescu-Rusescu" National Institute of Mother and Child Health, \\ Fetal Medicine Excellence Research Center, Bucharest, Romania; \\ ${ }^{8}$ Department of Surgery, "Pantelimon" Clinical Hospital, Bucharest, Romania; \\ ${ }^{9}$ Department of Internal Medicine, University Emergency Hospital Bucharest, Bucharest, Romania; \\ ${ }^{10}$ Department of Internal Medicine, "Fundeni" Clinical Institute, Bucharest, Romania; \\ ${ }^{11}$ Department of Obstetrics and Gynecology, "Elias" Emergency Hospital, Bucharest, Romania; \\ ${ }^{12}$ Department of Surgery, "Ponderas" Academic Hospital, Bucharest, Romania
}

\begin{abstract}
Background/Aim: Gastroenteropancreatic neuroendocrine carcinomas represent poorly differentiated neoplasms with a high capacity of spreading inducing the development of distant metastases. In such cases debulking surgery seems to offer a good chance for survival especially in well and moderately differentiated lesions. The aim of this study was to report the case of a 48-year-old patient submitted to surgery for moderately differentiated neuroendocrine gastric carcinoma with distant metastases. Case Report: The patient was initially investigated for hematemesis and weight loss and was diagnosed with a lesser curvature gastric tumor in association with liver and peritoneal metastases. Due to the extent of the disease, the
\end{abstract}

This article is freely accessible online.

Correspondence to: Irina Balescu, "Ponderas" Academic Hospital, Bucharest, Nicolae Caramfil 85a Street, Romania. Tel: +40 724077709, e-mail: irina.balescu@ ponderas-ah.ro

Key Words: Neuroendocrine carcinoma, liver metastases, Krukenberg tumors, debulking surgery. patient was initially submitted to neoadjuvant chemotherapy followed by surgery with radical intent. At the time of surgery subtotal gastrectomy en bloc with total omentectomy, peritonectomy, cholecystectomy and atypical liver resection were performed. Moreover, the two ovaries presented large tumoral masses so total hysterectomy with bilateral adnexectomy was performed. The histopathological studies confirmed the presence of a moderately differentiated neuroendocrine gastric carcinoma with negative resection margins. Conclusion: Multiple visceral resections might be needed in order to maximize the debulking effort in metastatic gastric neuroendocrine carcinomas.

Derived from enterochromaffin (Kultchitski) cells, neuroendocrine tumors have been initially described by Oberdorfer in 1907 in order to define carcinoma-like tumors with a lower degree of aggressiveness when compared to adenocarcinomas which also associate to a particular biological behavior $(1,2)$. Gastroduodenal neuroendocrine tumors account for a relatively low number of cases of neuroendocrine tumors and usually exhibit various clinical, pathological and histological features; therefore, standard therapeutic strategies are not clearly defined (3). When it 
comes to gastric neuroendocrine tumors, it is estimated that they account for less than $2 \%$ of all gastric malignancies, an increasing incidence being reported in the last decades (3). This fact has been initially considered to be caused by a higher use of proton pump inhibitors (PPI) which secondarily increases the rates of atrophic gastritis; although attractive, this theory could not be demonstrated in humans; only in rodents a direct correlation has been thus far established $(4,5)$. According to their characteristics, gastric neuroendocrine tumors can be classified in three types: type 1 - accounting for up to $80 \%$ of all lesions they are usually diagnosed in early stages of the disease, rarely metastasize and associate an overall good prognostic, type 2 lesions - accounting for up to $5 \%$ of cases - usually as part of type 1 multiple endocrine neoplasia - they are also usually diagnosed in early stages of the disease and rarely metastasize and type 3 tumors accounting for up to $15 \%$ of cases they are highly aggressive lesions with high risks of dissemination and requiring an aggressive surgical treatment (3). Unlike type 1 and 2, type 3 tumors are not associated with elevated gastric levels but are frequently associated with 5 hydroxytryptophan synthesis and consecutive carcinoid syndrome (6); moreover, type 3 tumors are usually located at the level of the antrum, usually measure more than $2 \mathrm{~cm}$ in diameter and might be associated with liver metastases (3). In consequence, type 3 lesions are associated with the poorest outcome, the five-year overall survival rate being usually lower than $33 \%$ (3).

\section{Case Report}

The 48-year-old patient with no significant medical history was initially investigated for diffuse epigastric pain, weight loss and hematemesis and was diagnosed at that moment with a lesser curvature gastric tumor.

Upper gastroscopy initially identified the presence of a $7 \times 6$ $\mathrm{cm}$ ulcerated lesion at the level of the lesser curvature which was biopsied, and histopathological studies revealed the presence of a G2 (moderately differentiated) gastric neuroendocrine tumor with relatively low levels of Ki67 of $12 \%$. The patient was further staged by performing a computed tomography which demonstrated the presence of diffuse peritoneal lesions of carcinomatosis, as well as the presence of five suspected liver lesions measuring between 2 and $5 \mathrm{~cm}$ at the level of segments II, IV, V, VI and VII (one at the level of each segment) and bilateral adnexal masses measuring $4 \times 4 \mathrm{~cm}$ (the left adnexa) and $6 \times 4 \mathrm{~cm}$ (the right adnexa). In the meantime, biological studies demonstrated higher levels of chromogranin A (232 IU/l) with normal ranges of gastrin, 5 hydroxyindol acetic acid, and no signs of anemia. Due to the extent of the lesions, the patient was initially submitted to neoadjuvant chemotherapy consisting of six cycles of cisplatin/etoposide. One month after ending the neoadjuvant chemotherapy, the patient was submitted to debulking surgery consisting of subtotal gastrectomy with gastrojejunal anastomosis, parietal and diaphragmatic peritonectomy, total omentectomy, cholecystectomy, atypical liver resection - segments II, IV, V, VI and VII and total hysterectomy with bilateral adnexectomy (Figures 1-4). Postoperative evolution was uneventful, and the patient was discharged in the eighth postoperative day. The histopathological studies confirmed the presence of a moderately differentiated gastric neuroendocrine carcinoma with negative resection margins of all the resected specimens.

\section{Discussion}

Type 3 gastric neuroendocrine tumors are associated with poor outcomes, and an aggressive treatment is needed in order to control the disease (3). In the study conducted by Kim et al. the authors underlined the fact that in such cases the overall prognosis is significantly influenced by the radicality of the surgical approach, the presence of distant metastases as well as by the development of recurrent disease (7).

In cases diagnosed with oligometastatic disease, the national comprehensive cancer network guidelines recommend debulking surgery whenever possible; however, in certain cases the extent of disease make surgery with curative intent impossible $(8,9)$. Therefore, in such cases neoadjuvant treatment followed by surgery might be needed; moreover, in cases in which association of gastrin synthesis is demonstrated, somatostatin analogues might be used in order to decrease the risk of recurrence $(10,11)$.

When it comes to the risk of developing Krukenberg tumors, this fact can be considered as common in patients with gastric cancer; however, ovarian metastases have been frequently described in gastric adenocarcinomas while in cases with other histopathological subtypes this finding is very uncommon. For example, in cases diagnosed with gastric neuroendocrine tumors ovarian metastases have been reported for the first time in a paper published in 2016 (6). In this study the authors presented the case of a 35-year-old patient diagnosed with early infiltrating cervical adenocarcinoma who was initially submitted to radical trachelectomy and sentinel node dissection; however, during the staging protocol she was diagnosed with solid, heterogeneous lesions at the level of both the ovaries suggesting the presence of benign masses. However, the ovarian biopsy raised the suspicion of malignant transformation while the immunohistochemical studies confirmed the presence of a low-grade neuroendocrine tumor with gastro-intestinal origin. Further on the upper digestive endoscopy confirmed the presence of a non-gastrin producing, moderately differentiated gastric neuroendocrine 


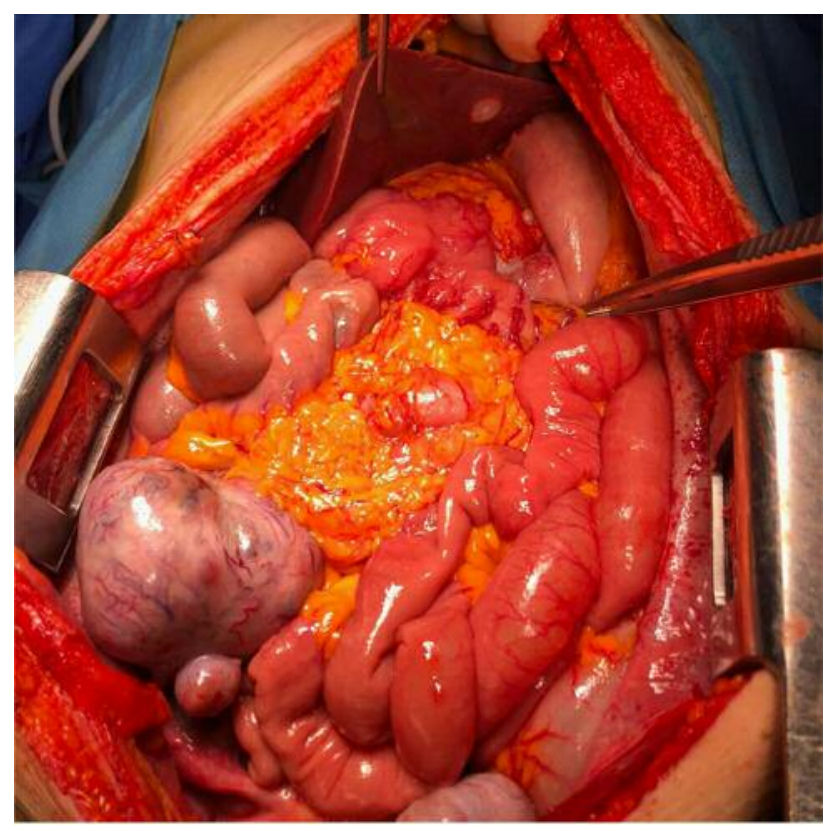

Figure 1. Initial intraoperative aspect - antral tumor in association with liver and ovarian metastases.

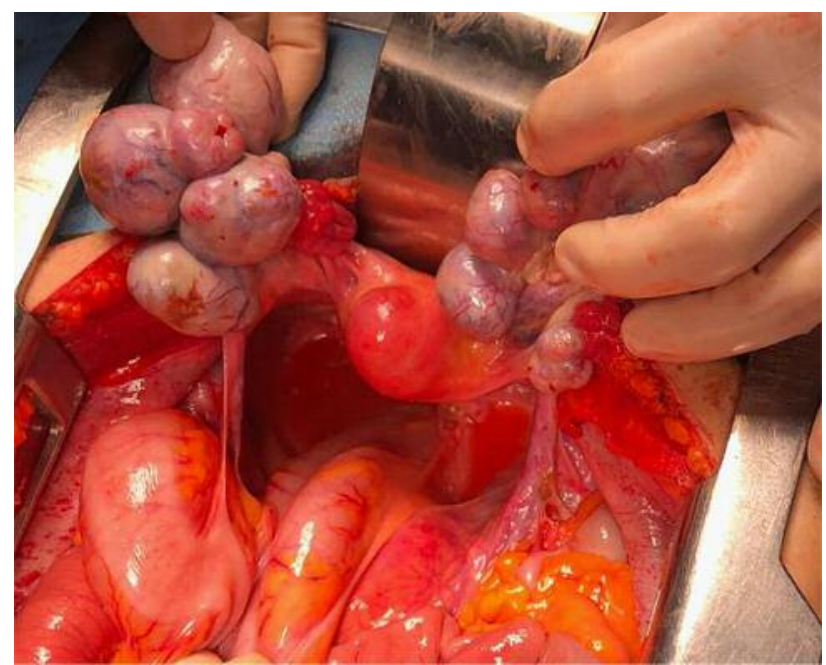

Figure 2. Intraoperative aspect - the two adnexa are transformed into tumoral masses - the aspect after mobilization.

carcinoma; the disease was finally staged after performing a positron emission tomography which demonstrated the presence of gastro-hepatic lymph node metastases in association with ovarian metastases. The patient was submitted to surgery with curative intent; in the meantime, the serum levels of chromogranin A normalized after surgery.

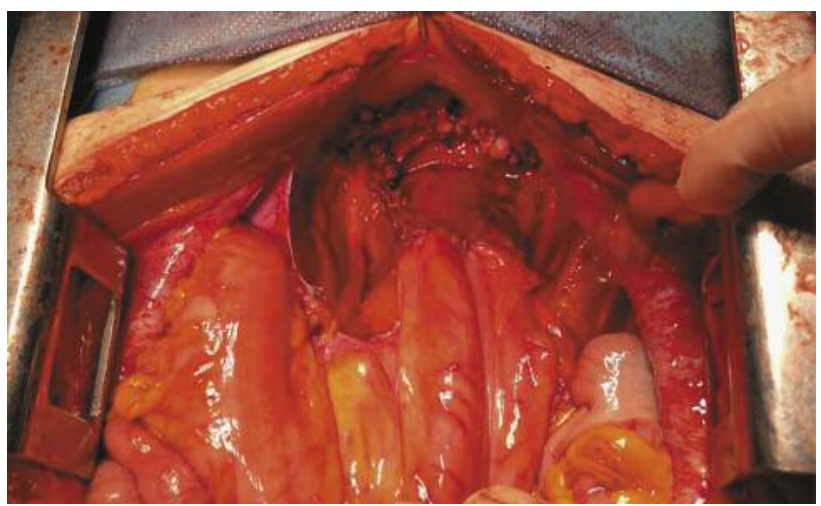

Figure 3. The aspect after total hysterectomy with bilateral adnexectomy.

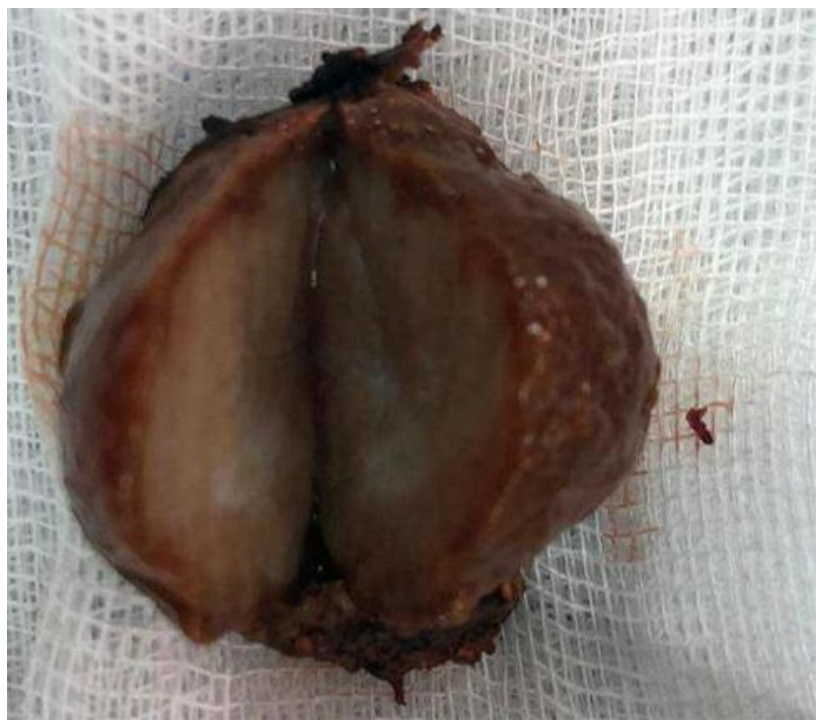

Figure 4. Metastasectomy for gastric neuroendocrine tumor liver metastasis.

Due to the absence of residual disease no adjuvant therapy was associated (6).

As for the association between neuroendocrine tumors and Krukenberg metastases, a study conducted by Limbach et al. underlined the fact that among 242 patients diagnosed with neuroendocrine tumors 27 cases developed Krukenberg tumors, $96.3 \%$ of them originating from small-bowel neuroendocrine lesions; moreover, the authors also underlined the fact that the presence of Krukenberg tumors was significantly associated with the presence of peritoneal carcinomatosis and surprisingly, with the presence of ureteral obstruction (12). Similarly to these findings, in our case the 
presence of Krukenberg tumors was associated with the presence of peritoneal carcinomatosis; however, interestingly, in our case the location of the largest peritoneal nodules was represented by the upper abdomen and not by the pelvic area.

Another issue that should be analyzed in regard to the case we presented is represented by the effectiveness of liver metastases resection. Most often patients presenting liver metastases from neuroendocrine tumors have small bowel or pancreatic primaries; as for cases diagnosed with liver metastases from gastric neuroendocrine primaries, more than two thirds will develop synchronous lesions and will originate from poorly differentiated, type 3 gastric tumors (6, 13). However, the presence of liver metastases in the setting of gastric neuroendocrine neoplasms represents an important predictor for the long-term outcomes $(14,15)$. In this particular case that we presented the development of synchronous liver metastases in a moderately differentiated gastric neuroendocrine tumor is rather the proof of prolong, indolent course of the disease. Moreover, when it comes to the feasibility of resection in such cases, surgery with curative intent is rarely possible in the setting of liver metastases (16-19). In order to create a universally accepted manner of treatment of gastric neuroendocrine tumors liver metastases, the European Neuroendocrine Tumor Society Consensus stated that in such cases three different patterns of disease spread to the liver exist, and according to the pattern of spread, specific management should be considered. The first pattern refers to the presence of liver metastases in a single lobe or in two adjacent segments; in such cases anatomic resection is feasible and recommended; the second pattern refers to cases presenting liver metastases predominantly in one lobe with satellite lesions in the other lobe; in such cases resection and destruction can be taken in consideration. The third pattern of spread refers to cases presenting diffuse multifocal metastases located in both the liver lobes; in such cases surgery should not be performed as a first intention procedure $(20,21)$. According to this recommendation, in the case we reported we decided for neoadjuvant chemotherapy; at the end of this treatment a partial diminishment of the metastatic lesions was encountered; therefore, we decided to perform surgery with curative intent once the disease was controlled by the administration of systemic therapy.

\section{Conclusion}

In patients diagnosed with type-3 gastric neuroendocrine tumors distant metastases can be encountered from the moment of initial diagnosis. In such cases, due to the extent of the disease and due to the pattern of liver involvement surgery might not be taken in consideration as first-line therapy. However, after completing the neoadjuvant therapy, debulking surgery with curative intent should be tempted. Even though, in certain cases multiple resections might be needed in order to achieve complete resection.

\section{Conflicts of Interest}

The Authors have no conflicts of interest to declare regarding this study.

\section{Authors' Contributions}

NB, AN, AF performed the surgical procedure; IH, Ciprian B reviewed literature data; $\mathrm{CD}, \mathrm{LI}, \mathrm{CP}$ realized the preoperative investigation of the patient; IB, Cristian B prepared the draft of the manuscript; IB was advisor of the surgical oncology procedures; NB, DC reviewed the final version of the manuscript. All Authors read and approved the final version of the manuscript.

\section{Acknowledgements}

This work was supported by the project entitled "Multidisciplinary Consortium for Supporting the Research Skills in Diagnosing, Treating and Identifying Predictive Factors of Malignant Gynecologic Disorders", project number PN-III-P1-1.2-PCCDI2017-0833.

\section{References}

1 Modlin IM, Moss SF, Chung DC, Jensen RT and Snyderwine E: Priorities for improving the management of gastroenteropancreatic neuroendocrine tumors. J Natl Cancer Inst 100(18): 1282-1289, 2008. PMID: 18780869. DOI: 10.1093/jnci/djn275

2 Modlin IM, Moss SF, Oberg K, Padbury R, Hicks RJ, Gustafsson BI, Wright NA and Kidd M: Gastrointestinal neuroendocrine (carcinoid) tumours: current diagnosis and management. Med J Aust 193(1): 46-52, 2010. PMID: 20618115.

3 O'Toole D, Delle FG and Jensen RT: Gastric and duodenal neuroendocrine tumours. Best Pract Res Clin Gastroenterol 26(6): 719-735, 2012. PMID: 23582915. DOI: 10.1016/j.bpg.2013.01.002

4 Ono M, Sato H, Kazumori H, Yuki M, Rumi MA, Ortega-Cava CF, Ishihara Y, Ishihara S, Adachi K and Kinoshita Y: Effect of a gastrin/cholecystokinin B receptor antagonist, S-0509, on the omeprazole-induced proliferation of gastric mucosa in rats. $\mathrm{J}$ Lab Clin Med 142(6): 364-371, 2003. PMID: 14713888. DOI: 10.1016/S0022-2143(03)00151-3

5 Hodgson N, Koniaris LG, Livingstone AS and Franceschi D: Gastric carcinoids: a temporal increase with proton pump introduction. Surg Endosc 19(12): 1610-1612, 2005. PMID: 16211437. DOI: $10.1007 / \mathrm{s} 00464-005-0232-4$

6 Manneh R, Castellano D, Caso O, Loinaz C, Jimenez J, Estenoz J, Calatayud M, Sepulveda JM and Garcia-Carbonero R: Welldifferentiated grade 2, type 3 gastrointestinal neuroendocrine tumour with bilateral metastatic ovarian involvement: report of an unusual case. Case Rep Oncol 9(1): 255-261, 2016. PMID: 27239181. DOI: $10.1159 / 000445940$

7 Kim BS, Oh ST, Yook JH, Kim KC, Kim MG, Jeong JW and Kim BS: Typical carcinoids and neuroendocrine carcinomas of the stomach: differing clinical courses and prognoses. Am J Surg 200(3): 328-333, 2010. PMID: 20385369. DOI: 10.1016/ j.amjsurg.2009.10.028 
8 Strosberg J, Nasir A, Cragun J, Gardner N and Kvols L: Metastatic carcinoid tumor to the ovary: a clinicopathologic analysis of seventeen cases. Gynecol Oncol 106(1): 65-68, 2007. PMID: 17475313. DOI: 10.1016/j.ygyno.2007.02.034

9 Pavel M, Grossman A, Arnold R, Perren A, Kaltsas G, Steinmuller T, de Herder W, Nikou G, Plockinger U, Lopes JM, Sasano H, Buscombe J, Lind P, O'Toole D and Oberg K: ENETS consensus guidelines for the management of brain, cardiac and ovarian metastases from neuroendocrine tumors. Neuroendocrinology 91(4): 326-332, 2010. PMID: 20453466. DOI: $10.1159 / 000287277$

10 Manfredi S, Pagenault M, Lajarte-Thirouard AS and Bretagne JF: Type 1 and 2 gastric carcinoid tumors: long-term follow-up of the efficacy of treatment with a slow-release somatostatin analogue. Eur J Gastroenterol Hepatol 19(11): 1021-1025, 2007. PMID: 18049175. DOI: 10.1097/MEG.0b013e328220eae0

11 Sideris L, Dube P and Rinke A: Antitumor effects of somatostatin analogs in neuroendocrine tumors. Oncologist 17(6): 747-755, 2012. PMID: 22628056. DOI: 10.1634/theoncologist.2011-0458

12 Limbach K, Pommier S, Dewey E, Leon E and Pommier R: Neuroendocrine metastases to the ovaries are significantly associated with small bowel neuroendocrine tumors and carcinomatosis. Am J Surg pii: S0002-9610(20)30118-5, 2020. PMID: 32145918. DOI: 10.1016/j.amjsurg.2020.02.040

13 Frilling $\mathrm{A}$ and Clift AK: Therapeutic strategies for neuroendocrine liver metastases. Cancer 121(11): 1172-1186, 2015. PMID: 25274401. DOI: 10.1002/cncr.28760

14 Ahmed A, Turner G, King B, Jones L, Culliford D, McCance D, Ardill J, Johnston BT, Poston G, Rees M, Buxton-Thomas M, Caplin $\mathrm{M}$ and Ramage JK: Midgut neuroendocrine tumours with liver metastases: results of the UKINETS study. Endocr Relat Cancer 16(3): 885-894, 2009. PMID: 19458024. DOI: 10.1677/ERC-09-0042

15 Pape UF, Berndt U, Muller-Nordhorn J, Bohmig M, Roll S, Koch M, Willich SN and Wiedenmann B: Prognostic factors of long-term outcome in gastroenteropancreatic neuroendocrine tumours. Endocr Relat Cancer 15(4): 1083-1097, 2008. PMID: 18603570. DOI: $10.1677 /$ ERC-08-0017
16 Marques B, Martins RG, Tralhao G, Couto J, Saraiva S, Ferrao H, Ribeiro J, Santos J, Martins T, Cadime AT and Rodrigues F: Gastric neuroendocrine neoplasm with late liver metastasis. Endocrinol Diabetes Metab Case Rep 2018: 2018. PMID: 30083348. DOI: 10.1530/EDM-18-0048

17 Dima SO, Dumitrascu T, Pechianu C, Grigorie RT, Brasoveanu V, Sorop A, Lupescu I, Purnichescu-Purtan R, Croitoru A, Bacalbasa N, Tanase A, Tomescu DR, Herlea V and Popescu I: Prognostic factors in patients with surgical resection of pancreatic neuroendocrine tumours. Acta Endocrinol (Buchar) 14(3): 389393, 2018. PMID: 31149288. DOI: 10.4183/aeb.2018.389

18 Mirancea GV, Morosanu AM, Carniciu S, Dima S, Bacalbasa N, Popescu I, Ionescu-Tirgoviste C and Mirancea N: Relevant infrastructural alterations in a pancreatic neuroendocrine tumor: an insulinoma case. Rom J Morphol Embryol 55(2 Suppl): 659668, 2014. PMID: 25178341.

19 Bacalbasa N, Balescu I, Dima S and Popescu I: Hepatic resection for liver metastases from cervical cancer is safe and may have survival benefit. Anticancer Res 36(6): 3023-3027, 2016. PMID: 27272821.

20 Frilling A, Sotiropoulos GC, Li J, Kornasiewicz O and Plockinger U: Multimodal management of neuroendocrine liver metastases. HPB (Oxford) 12(6): 361-379, 2010. PMID: 20662787. DOI: $10.1111 / \mathrm{j} .1477-2574.2010 .00175 . x$

21 Palumbo C, Pecoraro A, Knipper S, Rosiello G, Tian Z, Shariat SF, Simeone C, Briganti A, Saad F, Berruti A, Antonelli A and Karakiewicz PI: Survival and complication rates of metastasectomy in patients with metastatic renal cell carcinoma treated exclusively with targeted therapy: a combined population-based analysis. Anticancer Res 39(8): 4357-4361, 2019. PMID: 31366530. DOI: 10.21873 /anticanres.13604

Received March 9, 2020

Revised March 24, 2020

Accepted March 26, 2020 